\title{
Nouvelles du corps médical
}

\author{
Todesfälle / Décès / Decessi \\ Heinrich König (1921), † 12.12.2020, \\ Facharzt für Ophthalmologie, \\ 3205 Gümmenen
}

\section{Rolf Marc Anner (1943), † 1.1.2021, \\ 1232 Confignon}

Heinz Stefan Herzka (1935), † 14.2.2021,

Facharzt für Kinder- und Jugendpsychiatrie und -psychotherapie, 8006 Zürich

François Clément (1929), † 5.7.2021,

Spécialiste en hématologie et Spécialiste en médecine interne générale,

1005 Lausanne

Joseph Rossier (1935), † 6.7.2021,

Spécialiste en médecine interne générale, 3968 Veyras

\section{Praxiseröffnungen / \\ Nouveaux cabinets médicaux / \\ Nuovi studi medici}

BE

Greta Frick, Fachärztin für Allgemeine Innere Medizin, FMH, Elfenauweg 11, 3006 Bern

VD

Maxime Grosclaude, Spécialiste en médecine physique et réadaptation, $\mathrm{FMH}$, Chemin des Saules 4A, 1260 Nyon

Ärztegesellschaft des Kantons Bern Ärztlicher Bezirksverein Bern Regio Zur Aufnahme als ordentliches Mitglied hat sich angemeldet:

Karin N. Sauter, Fachärztin für Pathologie, FMH, Pathologie Länggasse, Worblentalstrasse 32 West, 3063 Ittigen

Einsprachen gegen dieses Vorhaben müssen innerhalb 14 Tagen seit der Veröffentlichung schriftlich und begründet bei den Co-Präsidenten des Ärztlichen Bezirksvereins Bern
Regio eingereicht werden. Nach Ablauf der Frist entscheidet der Vorstand über die Aufnahme der Gesuche und über allfällige Einsprachen.

\section{Ärztegesellschaft des Kantons Luzern \\ Zur Aufnahme in unsere Gesellschaft Sektion Stadt haben sich gemeldet:}

Le Phung Banh, Fachärztin für Gynäkologie und Geburtshilfe, Frauenarztpraxis Grendel, Grendelstrasse 21, 6004 Luzern

Maria Helfenstein, Fachärztin für Ophthalmologie, FMH, ab 1.9.2021: Augenärzte Belvista, Stadthausstrasse 1, 6003 Luzern

Andrea Katharina Müller, Fachärztin für Chirurgie, ab 1.8.2021: Centramed Luzern, Frankenstrasse 2, 6002 Luzern

Sebastian Zaremba, Facharzt für Neurologie, Klinik für Schlafmedizin Luzern, Lützelmattstrasse 3, 6006 Luzern

Einsprachen sind innert 20 Tagen nach der Publikation schriftlich und begründet zu richten an: Ärztegesellschaft des Kantons Luzern, Schwanenplatz 7, 6004 Luzern

Zur Aufnahme in unsere Gesellschaft Sektion Gäu hat sich gemeldet:

Stefan Görnitz, Praktischer Arzt, Surenweidpraxis, Surenweidstrasse 1, 6208 Oberkirch

Einsprachen sind innert 20 Tagen nach der Publikation schriftlich und begründet zu richten an: Ärztegesellschaft des Kantons Luzern, Schwanenplatz 7, 6004 Luzern

Ärztegesellschaft des Kantons Schwyz Zur Aufnahme in die Ärztegesellschaft des Kantons Schwyz haben sich angemeldet:

Monica Costache, Praktische Ärztin, Übernahme Praxis Dr. Langer in Steinen zum 1.9.2021
Thomas Degen, Facharzt für Allgemeine Innere Medizin, FMH, Leitender Arzt Innere Medizin am Spital Lachen

Michael Muffler, Facharzt für Allgemeine Innere Medizin, FMH, ab Mitte Juli 2021 Ärztezentrum Reichenburg SZ

Valentina Petroulia, Fachärztin für Radiologie, ab 1.8.2021 Stv. Chefärztin Röntgeninstitut Schwyz

Einsprachen gegen diese Aufnahmen richten Sie schriftlich innert 20 Tagen an Dr. med. Uta Kliesch, Maria-Hilf-Strasse 9, 6430 Schwyz, oder per Mail an uta.kliesch[at]hin.ch

Gesellschaft der Ärztinnen und Ärzte des Kantons Solothurn

Zur Aufnahme als ordentliche Mitglieder haben sich angemeldet:

Kristina Affolter, Fachärztin für Allgemeine Innere Medizin und Fachärztin für Pneumologie, FMH, Kantonsspital Olten, Pneumologie, Baslerstr. 150, 4600 Olten

Christine Azar, Fachärztin für Psychiatrie und Psychotherapie, FMH, Solopsy, Werkhofstr. 2, 4500 Solothurn

Marc Fischer, Facharzt für Allgemeine Innere Medizin und Facharzt für Kardiologie, FMH, Herzpraxis Olten, Tannwaldstr. 2, 4600 Olten

Mahire Krüger, Fachärztin für Gynäkologie und Geburtshilfe, Gesundheitszentrum Monvia, Frohburgstr. 4, 4600 Olten

Ulrich Lachmund, Facharzt für Ophthalmologie und Facharzt für Radiologie, FMH, Augenzentrum Oensingen, Sternenweg 16, 4702 Oensingen

Stephan Ness, Facharzt für Anästhesiologie, FMH, rentanesth AG, Bahnhofstr. 17, 5000 Aarau 
Andreas Weinberger, Facharzt für Ophthalmologie, FMH, Pallas Kliniken AG, Louis-GiroudStr. 20, 4600 Olten

Markus Wopmann, Facharzt für Kinderund Jugendmedizin, FMH, MediService AG, Ausserfeldweg 1, 4528 Zuchwil

Einsprachen gegen diese Aufnahmen sind innerhalb 14 Tagen nach der Publikation schriftlich und begründet bei den Co-Präsidenten der Gesellschaft der Ärztinnen und Ärzte des Kantons Solothurn, GAeSO,

Postfach 332, 4502 Solothurn, einzureichen.
Ärztegesellschaft Thurgau

Die Ärztegesellschaft Thurgau informiert über folgende Neuanmeldungen:

Eva-Maria-Charlotte Falkenberg, Fachärztin für Allgemeine Innere Medizin, Sonnenstrasse 9a, 8572 Berg

Peter Frischknecht,

Facharzt für Allgemeine Innere Medizin,

Poststrasse 28, 9220 Bischofszell

Nazmi Krasniqi, Facharzt für Kardiologie, FMH, Katzenrütistrasse 81A, 8153 Rümlang

Regina Maria Paula Kugler, Fachärztin für

Kinder- und Jugendmedizin, Wiesental 21, 9312 Häggenschwil

\section{Sujets d'actualité en ligne}

www.bullmed.ch $\rightarrow$ Tour d'horizon
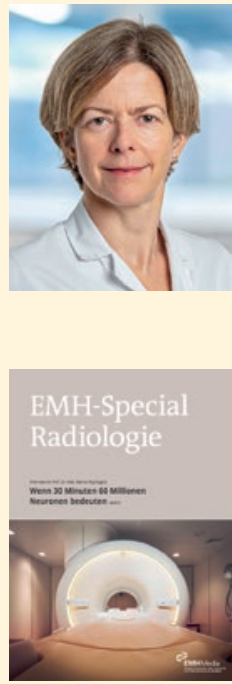

Entretien avec Prof. Dr méd. Maria Wertli, présidente Commission qualité de la Société Suisse de Médecine Interne Générale (SSMIG)

\section{Indicateurs de qualité - Avantages et limites}

La Commission qualité de la SSMIG présente pour la première fois les nouveaux indicateurs de qualité pour le traitement stationnaire.

Editorial de Sandra Ziegler, directrice des EMH Editions médicales suisses

\section{EMH-Special: une nouvelle série de publications}

Des articles sur des sujets captivants, de précieuses informations pour la pratique médicale - voici ce qui caractérise la nouvelle série de publications «EMH-Special». 